\title{
POLYNOMIALS WITH MINIMAL VALUE SET OVER GALOIS RINGS
}

\section{MARIA T. ACOSTA-DE-OROZCO}

Department of Mathematics

Penn State University

Beaver Campus

Monaca, Pennsylvania 15061

\section{and \\ JAVIER GOMEZ-CALDERON}

Department of Mathematics

Penn State University

New Kensington Campus

New Kensington, Pennsylvania 15068

(Received September 12, 1989)

\begin{abstract}
Let $G R\left(p^{n}, m\right)$ denote the Galois ring of order $p^{n m}$, where $p$ is a prime. In this paper we define and characterize minimal value set polynomials over $G R\left(p^{n}, m\right)$.

KEY WORDS AND PHRASES. Minimal polynomials, Galois rings, special value set polynomials over Galois ring.

1985 REVISED AMS SUBJECT CLASSIFICATION CODE. Primary 11 T06.
\end{abstract}

1. INTRODUCTION. Let $G F(q)$ denote the finite field of order $q$ where $q$ is a prime power. If $f(x)$ is a polynomial of positive degree $d$ over $G F(q)$, let $V(f)=\{f(x): x \in G F(q)\}$ denote the image or value set of $f(x)$ and let $|V(f)|$ denote the cardinality of $V(f)$. Since a polynomial of degree $d$ cannot assume a given value more than $d$ times over any field, it is clear that

$$
[(q-1) / d]+1 \leq|V(f)|
$$

where $[x]$ denotes the greatest integer $\leq x$.

A polynomial for which equality is achieved in (1.1) is called a minimal value set polynomial. Minimal value set polynomials over finite fields have been studied in Carlitz, Lewis, Mills and Straus [1], and Mills [4]. Among their results, they proved that if $|V(f)| \geq 3$ and $2<d<p, p$ the characteristic of $G F(q)$, then $d$ divides $q-1$ and $f(x)$ is of the form

$$
f(x)=a(x-b)^{d}+c, \quad a \neq 0
$$


Conversely, if $d$ divides $q-1$ and $f(x)$ is of this form, then

$$
|V(f)|=[(q-1) / d]+1
$$

In the present paper we define and study polynomials with minimal value set over Galois rings which are finite extensions of the ring $Z_{p^{n}}$ of integers modulo $p^{n}$ where $p$ is a prime and $n \geq 1$. In particular, $G R\left(p^{n}, m\right)$ will denote the Galois ring of order $p^{n m}$ which can be obtained as a Galois extension of $Z_{p^{n}}$ of degree $m$. Thus, $G R\left(p^{n}, 1\right)=Z_{p^{n}}$ and $G R(p, m)=G F\left(p^{m}\right)$, the finite field of order $p^{m}$. The reader can find further details concerning Galois rings in the reference [3].

We start obtaining a lower bound for the cardinality of value set polynomials over the Galois ring $G R\left(p^{n}, m\right)$. As it could be expected, our lower bound reduces to $[(q-1) / d]+1$ when $n=1$. More precisely, we have the following

2. MAIN RESULTS

LEMMA 2.1. Let $f(x)$ be a monic polynomial of degree $d$ over the Galois ring $G R\left(p^{n}, m\right)$, $n \geq 2$. Let $V(f)=\left\{f(x): x \in G R\left(p^{n}, m\right)\right\}$ denote the value set of $f(x)$ and let $|V(f)|$ denote the cardinality of $V(f)$. Let $q=p^{m}$. Assume $2<d<\min \{p, 3 \sqrt{2 q}\}$. Then

$$
[(q-1) / d] q^{n-1}+1 \leq|V(f)|
$$

where $[x]$ denotes the greatest integer $\leq x$.

The proof uses the following Lemma that is a generalization of a well known result about lifting solutions over $Z_{p} n$.

LEMMA 2.2. Let $f(x)$ be a monic polynomial with coefficients in $G R\left(p^{n}, m\right)$. Assume $n \geq 2$ and let $T$ be a solution of the equation $f(x)=0$ in the Galois ring $G R\left(p^{n-1}, m\right)$.

(a) Assume $f^{\prime}(T) \neq 0$ over the field $G R(p, m)$. Then $T$ can be lifted in a unique way from $G R\left(p^{n-1}, m\right)$ to $G R\left(p^{n}, m\right)$.

(b) Assume $f^{\prime}(T)=0$ over the field $G R(p, m)$. Then we have two possibilities:

(b.1) If $f(T)=0$ over $G R\left(p^{n}, m\right), T$ can be lifted from $G R\left(p^{n-1}, m\right)$ to $G R\left(p^{n}, m\right)$ in $p^{m}$ distinct ways.

(b.2) If $f(T) \neq 0$ over $G R\left(p^{n}, m\right), T$ cannot be lifted from $G R\left(p^{n-1}, m\right)$ to $G R\left(p^{n}, m\right)$.

PROOF. Let $T$ be a solution of the equation $f(x)=0$ in the ring $G R\left(p^{n-1}, m\right)$. Let $Q$ be an element of $G R(p, m)$. Then, by Taylor's formula

$$
f\left(T+Q p^{n-1}\right)=f(T)+f^{\prime}(T) Q p^{n-1}
$$

over the ring $G R\left(p^{n}, m\right)$. Further, since $f(T)=0$ over $G R\left(p^{n-1}, m\right)$,

$$
f\left(T+Q p^{n-1}\right)=\left[k+f^{\prime}(T) Q\right] p^{n-1}
$$

for some $k$ in $G R(p, m)$. Therefore, $f\left(T+Q p^{n-1}\right)=0$ over $G R\left(p^{n}, m\right)$ if and only if

$$
k+f^{\prime}(T) Q=0
$$

over the field $G R(p, m)$. Now, if $f^{\prime}(T) \neq 0$ then the linear equation $(*)$ has a unique solution $Q$ in 
$G R(p, m)$. On the other hand, if $f^{\prime}(T)=0,(*)$ has no solutions when $k \neq 0$, and $p^{m}$ solutions when $k=0$.

This completes the proof of the lemma.

PROOF OF LEMMA 2.1. Let $V(f)$ denote the value set of $f(x)$ over $G R\left(p^{n}, m\right)$. Let $\bar{f}(x)$ denote the reduction of $f(x)$ modulo $p$. Let $V(\bar{f})$ denote the value set of $\bar{f}(x)$ over the field $G R(p, m)$. For $\bar{b} \in V(\bar{f})$, let $L(\bar{b})$ denote the set of elements in $V(f)$ that reduce to $\bar{b}$ modulo $p$, i.e.

$$
L(\bar{b})=\{b \in V(f): b \equiv \bar{b}(\bmod p)\}
$$

So, it is clear that

$$
1 \leq|L(\bar{b})| \leq q^{n-1}
$$

Now, if $\bar{f}(x)-\bar{b}$ has at least one simple root $\bar{r}$ over the field $G R(p, m)=G F(q)$, then, by Lemma $2.2, \bar{r}$ can be lifted from $G R(p, m)$ to $G R\left(p^{n}, m\right)$ for all $b$ in $G R\left(p^{n}, m\right), b \equiv \bar{b}(\bmod p)$. Hence,

$$
|L(\bar{b})|=\left(p^{n-1}\right)^{m}=q^{n-1}
$$

Conversely, if $|L(\bar{b})|<q^{n-1}$ then $\bar{f}(x)-\bar{b}$ has no simple roots over the field $G F(q)$. We also observe that the number of images $\bar{b}$ such that $|L(\bar{b})|<q^{n-1}$ is as most $d-1$. Therefore,

$$
(|V(\bar{f})|-N) q^{n-1}+N \leq|V(f)| \leq|V(\bar{f})| q^{n-1}
$$

where $N$ denotes the number of images $\bar{b}$ such that $\bar{f}(x)-\bar{b}$ has no simple roots over the field $G F(q)$. So, $0 \leq N \leq d-1$.

Now, if $N \neq 1, \bar{f}(x)$ is not a minimal value set polynomial. Hence, according to [2], the value set of $\bar{f}(x)$ satisfies the inequality

$$
[(q-1) / d]+2(q-1) / d^{2} \leq|V(\bar{f})|
$$

Therefore,

$$
\left.([q-1) / d]+2(q-1) / d^{2}-(d-1)\right) q^{n-1}+(d-1) \leq|V(f)|
$$

for all $N, N \neq 1$.

On the other hand, for $N=1$ we have

$$
[(q-1) / d] q^{n-1}+1 \leq|V(f)|
$$

Now, it is easy to see that $d<3 \sqrt{2 q}$ implies

$$
[(q-1) / d] q^{n-1}+1<\left([(q-1) / d]+2(q-1) / d^{2}-(d-1)\right) q^{n-1}+(d-1) .
$$

This completes the proof of Lemma 2.1 .

DEFINITION. Let $f(x)$ be a monic polynomial of degree $d$ over the Galois ring $G R\left(p^{n}, m\right)$, 
$n \geq 2$. Let $q=p^{m}$. Assume $2<d<\min \{p, 3 \sqrt{2 q}\}$. Then $f(x)$ is called minimal value set polynomial if

$$
[(q-1) / d] q^{n-1}+1=|V(f)|
$$

We are ready for the main result of the paper.

THEOREM 1. Let $f(x)$ be a monic polynomial of degree $d$ over the Galois ring $G R\left(p^{n}, m\right)$, $n \geq 2$. Let $q=p^{m}$. Assume $2<d<\min \{p, 3 \sqrt{2 q}\}$. If $f(x)$ is a minimal value set polynomial, then $d \geq n, d$ divides $p-1$ and $f(x)$ is of the form

$$
f(x)=b_{o}+\left(\sum_{i=1}^{n-1} p^{n-i} b_{i}(x-a)^{i}\right)+p\left(\sum_{i=n}^{d-1} b_{i}(x-a)^{i}\right)+(x-a)^{d} .
$$

Conversely, if $d \geq n, d$ divides $p-1$ and $f(x)$ is of this form, then $f(x)$ is a minimal value set polynomial over $G R\left(p^{n}, m\right)$.

PROOF. With notation as in Lemma 2.1 , it is easy to see that $f(x)$ is a minimal value set polynomial over $G R\left(p^{n}, m\right)$ if and only if the following two conditions hold.

(i) $\bar{f}(x)$ is a minimal polynomial over the field $G R(p, m)=G F(q)$.

(ii). $\quad N=1$ and for this unique image $\bar{b}$ we have $L(\bar{b})=\{b\}$.

First, suppose $f(x)$ is a minimal value set polynomial over $G R\left(p^{n}, m\right)$. Let $\bar{r}$ be an element of $G R(p, m)$ so that $\bar{f}(\bar{r})=\bar{b}$. Then, by (ii), $f(\bar{r}+r p)=b$ for all $r$ in $G R\left(p^{n}, m\right)$. Thus, by Taylor's polynomial formula,

$$
f^{(i)}(\bar{r})=0
$$

over $G R\left(p^{n-i}, m\right)$ for $i=1, \ldots, n-1$. Hence, since $f(x)$ is monic, $d \geq n$ and $f(x)$ has the form given in (3). We also obtain, from (i), that $d$ divides $p-1$.

Now suppose $d \geq n, d$ divides $p-1$ and $f(x)$ is of the form given in (2.2). Then $f^{(i)}(a)=0$ over $G R\left(p^{n-i}, m\right)$ for $\imath=1, \ldots, n-1$. Thus, $f(a+p x)=0$ for all $x$ in $G R\left(p^{n}, m\right)$, from which condition (ii) follows. Finally, we obtain condition (i) by a straightforward application of [1].

This completes the proof of the theorem.

COROLLARY. With notation as in Theorem 1 , if $f(x)$ is a minimal yalue set polynomial over $G R\left(p^{n}, m\right)$, then $f(x)$ is a minimal value set polynomial over $G R\left(p^{i}, m\right)$ for $i=1,2, \ldots, n-1$.

\section{REFERENCES}

1. CARLITZ, L., LEWIS, D.J., MILLS, W.H. and STRAUS, E.G., Polynomials over finite fields with minimal value sets, $\underline{\text { Mathematika }} \underline{8}$ (1961), 121-130.

2. GOMEZ-CALDERON, J., A note on polynomials with minimal value set over finite fields, Mathematika $\underline{35}$ (1988), 144-148.

3. MCDONALD, B.R., "Finite Rings with Identity", Marcel Dekker, New York, 1974.

4. MILLS, W.H., Polynomials with minimal value sets, Pacific J. Math. 14 (1964), 225-241. 


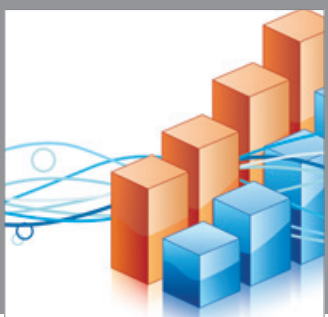

Advances in

Operations Research

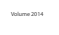

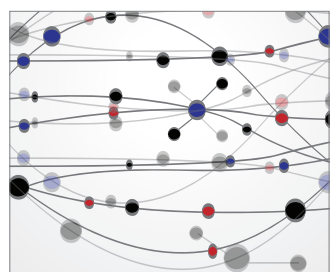

\section{The Scientific} World Journal
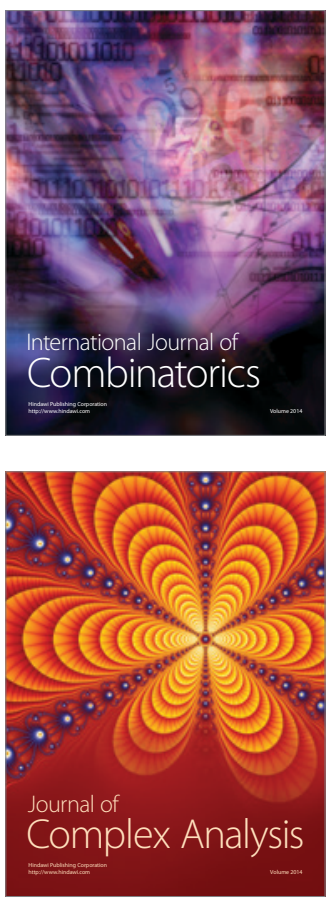

International Journal of

Mathematics and

Mathematical

Sciences
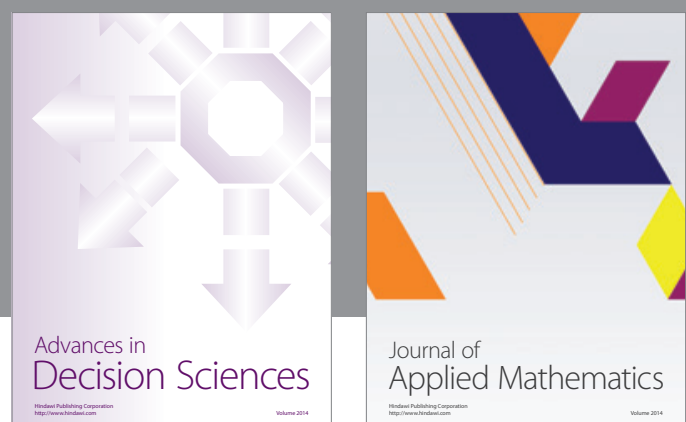

Journal of

Applied Mathematics
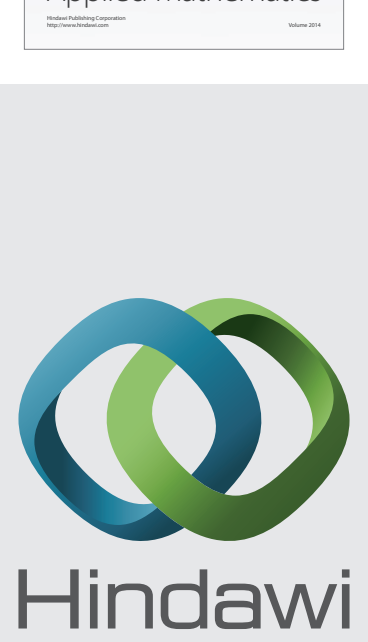

Submit your manuscripts at http://www.hindawi.com
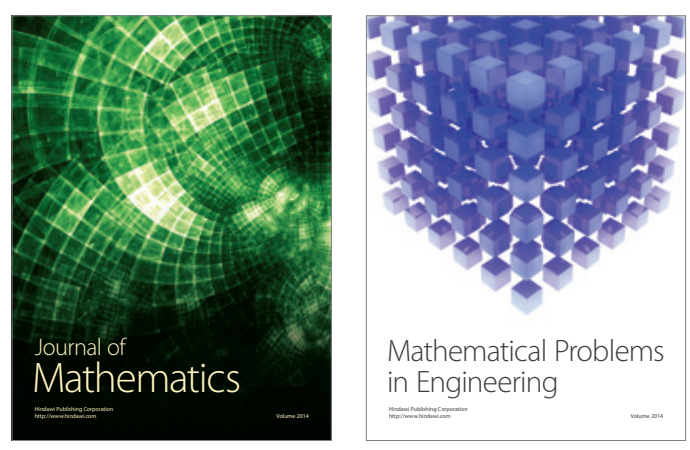

Mathematical Problems in Engineering
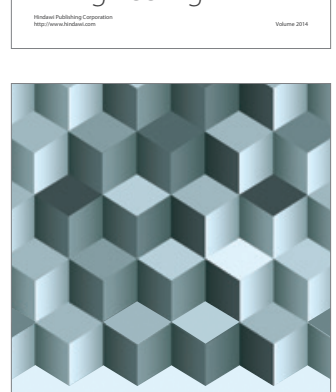

Journal of

Function Spaces
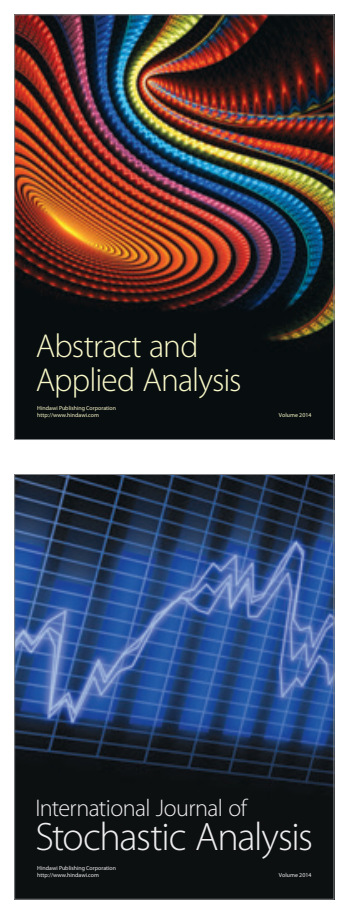

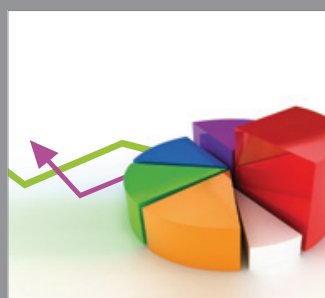

ournal of

Probability and Statistics

Promensencen
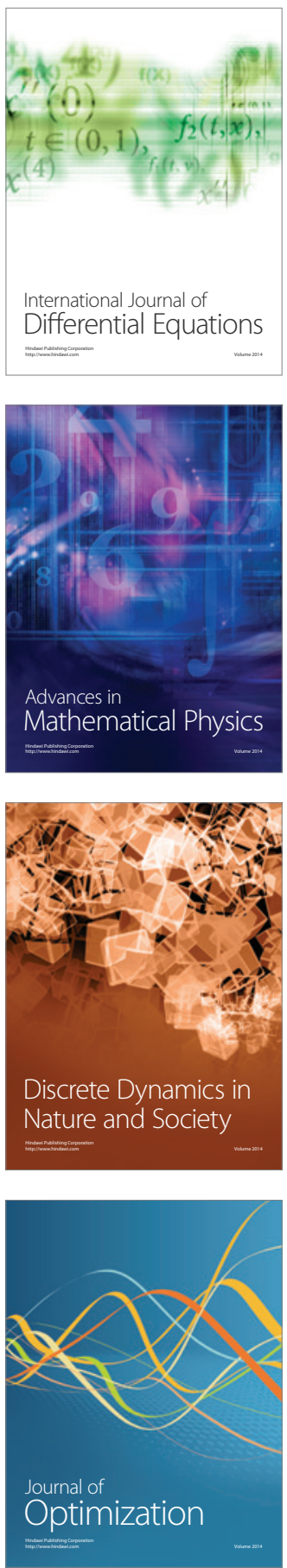